\title{
ON THE MARCINKIEWICZ ZYGMUND LAWS OF LARGE NUMBERS FOR NEGATIVELY DEPENDENT RANDOM FIELDS
}

\author{
Mi-HwA Ko
}

\begin{abstract}
In this paper we provide extensions of the Marcinkiewicz Zygmund laws of large numbers for i.i.d random variables with multidimensional indices to the case of negatively dependent random fields.
\end{abstract}

\section{Introduction}

Let $Z_{+}^{d}$, where $d$ is a positive integer, denote the positive integer $d$ dimensional lattice points. The notation $\mathbf{m} \leq \mathbf{n}$, where $\mathbf{m}=\left(m_{1}, m_{2}\right.$, $\left.\cdots, m_{d}\right)$ and $\mathbf{n}=\left(n_{1}, n_{2}, \cdots, n_{d}\right)$ in $\mathbb{Z}_{+}^{d}$, means that $m_{i} \leq n_{i}$ for all $1 \leq$ $i \leq d$. The following multiindex version of the Marcinkiewicz Zygmund strong laws of large numbers was given in Gut(1978).

Theorem A Let $0<r<2$, and suppose that $X,\left\{X_{\mathbf{i}}, \mathbf{i} \in \mathbb{Z}_{+}^{d}\right\}$ is a field of i.i.d random variables with partial sums $S_{\mathbf{n}}=\sum_{\mathbf{i} \leq \mathbf{n}} X_{\mathbf{i}}, \mathbf{i} \in Z_{+}^{d}$. If $E|X|^{r}\left(\log ^{+}|X|\right)^{d-1}<\infty$ and $E X=0$ when $1 \leq r<2$, then

$$
\frac{S_{\mathbf{n}}}{|\mathbf{n}|^{\frac{1}{r}}} \rightarrow 0 \text { a.s. as } \mathbf{n} \rightarrow \infty \text {. }
$$

Conversely, if (1.1) holds, then $E|X|^{r}\left(\log ^{+}|X|\right)^{d-1}<\infty$ and $E X=0$ when $1 \leq r<2$. Here $|\mathbf{n}|=\prod_{i=1}^{d} n_{i}$ and $\mathbf{n} \rightarrow \infty$ means $\min _{1 \leq i \leq d} n_{i} \rightarrow$ $\infty$, that is, all coordinates tend to infinity. Also, throughout the paper, $\log ^{+} x=\max \{1, \log x\}$.

Next, we turn to our attention to the negative dependence. Two random variables $X$ and $Y$ are negative quadrant dependent(NQD) if $P(X \leq x, Y \leq y) \leq P(X \leq x) P(Y \leq y)$ for all $x, y \in \mathbb{R}$. A finite family

Received March 3, 2011. Accepted March 15, 2011.

2000 Mathematics Subject Classification. 60F15, 60G50.

Key words and phrases. Marcinkiewicz Zygmund laws of large numbers; Almost sure convergence; Random field; Negative quadrant dependence, Negative association. 
$\left\{X_{1}, \cdots, X_{n}\right\}$ is said to be negatively associated(NA) if for any disjoint subsets $A, B \subset\{1, \cdots, n\}$ and any real coordinatewise nondecreasing functions $f$ on $\mathbb{R}^{A}, g$ on $\mathbb{R}^{B}, \operatorname{Cov}\left(f\left(X_{i}, i \in A\right), g\left(X_{j}, j \in B\right)\right) \leq 0$. An infinite family of random variables is negatively associated if every finite subfamily is NA. These concepts of negative dependences were introduced by Lehmann(1966) and Joag-Dev and Proschan(1983), respectively.

The notions of NQD and NA can be extended to the random variables with multidimensional indices.

Following the proof of Theorem 2 of Etemadi(1981), Matula(1992) proved the next strong law of large numbers for a field of identically distributed pairwise NQD random variables.

Theorem B Let $\left\{X_{\mathbf{i}}, \mathbf{i} \in \mathbb{Z}_{+}^{d}\right\}$ be a field of identically distributed pairwise NQD random variables. If $E\left|X_{1}\right|\left(\log ^{+}\left|X_{1}\right|\right)^{d-1}<\infty$ and $E X_{1}=0$, then

$$
\frac{S_{\mathbf{n}}}{|\mathbf{n}|} \rightarrow 0 \text { a.s. as } \mathbf{n} \rightarrow \infty \text {. }
$$

Since NA random variables are pairwise NQD random variables Theorem B still holds under NA assumption.

In the case of $d=1$, the Marcinkiewicz Zygmund strong law of large numbers for identically distributed NA random variables was given by Liu, Gan and Chen (1999) as follows.

Theorem $\mathbf{C}$ Let $0<r<2$, and $\left\{X_{n}, n \geq 1\right\}$ be a sequence of identically distributed NA random variables. If $E\left|X_{1}\right|^{r}<\infty$, and $E X_{1}=0$ when $1 \leq r<2$, then

$$
\frac{S_{n}}{n^{\frac{1}{r}}} \rightarrow 0 \text { a.s. as } n \rightarrow \infty \text {. }
$$

Conversely if almost sure convergence holds as stated, then $E\left|X_{1}\right|^{r}<\infty$ when $1 \leq r<2$.

In this paper, we extend Theorem A to the negative dependent random variables with multidimensional indices as well as generalize Theorem $\mathrm{C}$ to the multidimensional case.

For convenience we use the parametrization $\alpha=\frac{1}{r}$ i.e., $1 \leq r<2$ is translated into $\frac{1}{2}<\alpha \leq 1$ in Section 2 . 


\section{Main results}

A first important observation is that inequalities which do not depend on the (partial) order of the index set $\mathbb{Z}_{+}^{d}$, such as the triangle inequality, moment inequalities for sums, and so on remain valid automatically. Namely, such relations only depend on the fact that if $\left\{X_{\mathbf{k}}, \mathbf{k} \in \mathbb{Z}_{+}^{d}\right\}$ are random variables and $\left\{S_{\mathbf{n}}, \mathbf{n} \in \mathbb{Z}_{+}^{d}\right\}$ their partial sums, then $S_{\mathbf{n}}$ is simply a sum of $|\mathbf{n}|$ random variables.

The following quantities and their asymptotic behavior will be crucial to prove our main results. Let

$$
g(\nu)=\operatorname{card}\{\mathbf{n}:|\mathbf{n}| \leq \nu\} \text { and } \Delta g(\nu)=\operatorname{card}\{\mathbf{n}:|\mathbf{n}|=\nu\}=\sum_{|\mathbf{n}|=\nu} 1 .
$$

The following asymptotics hold:

$$
\frac{g(\nu)}{\nu(\log \nu)^{d-1}} \rightarrow \frac{1}{(d-1) !} \text { as } \nu \rightarrow \infty .
$$

See Hardy(1954), Chapter XVIII(for the case $d=2$; the general case is analogous) and Titchmarsh(1951), Chapter 12.

Another important observation is that, since all terms we consider are nonnegative, we may change the order of summation, in particular as follows(cf. Gut $(1978,1980))$

$$
\sum_{\mathbf{n}} \cdots=\sum_{\nu \geq 1} \sum_{|\mathbf{n}|=\nu} \cdots
$$

More importantly, whenever the functions involving $\mathbf{n}$ only depend on the value of $|\mathbf{n}|$, the second summation can be simplified further. For example, we have

$$
\begin{aligned}
\sum_{\mathbf{n}} \frac{1}{|\mathbf{n}|} P\left(\left|X_{\mathbf{k}}\right|>\epsilon|\mathbf{n}|^{\frac{1}{p}}\right) & =\sum_{\nu \geq 1} \sum_{|\mathbf{n}|=\nu} \frac{1}{|\mathbf{n}|} P\left(\left|X_{\mathbf{k}}\right| \geq \epsilon|\mathbf{n}|^{\frac{1}{p}}\right) \\
& =\sum_{\nu \geq 1} g(\nu) \frac{1}{\nu} P\left(\left|X_{\mathbf{k}}\right| \geq \epsilon \nu^{\frac{1}{p}}\right)
\end{aligned}
$$

(see Gut and Spătăru(2003)). This observation should be kept in mind thr oughout.

Theorem 2.1 Let $\frac{1}{2}<\alpha \leq 1$ and $\left\{X_{\mathbf{i}}, \mathbf{i} \in \mathbb{Z}_{+}^{d}\right\}$ be a field of identically distributed negatively associated random variables with $E X_{1}=0$. Then 
the following statements are equivalent:

$$
\begin{gathered}
E\left|X_{\mathbf{1}}\right|^{\frac{1}{\alpha}}\left(\log ^{+}\left|X_{\mathbf{1}}\right|\right)^{d-1}<\infty \text { and } E X_{\mathbf{1}}=0, \\
\frac{S_{\mathbf{n}}-E S_{\mathbf{n}}}{|\mathbf{n}|^{\alpha}} \rightarrow 0 \text { a.s. as } \mathbf{n} \rightarrow \infty,
\end{gathered}
$$

where $S_{\mathbf{n}}=\sum_{\mathbf{1} \leq \mathbf{i} \leq \mathbf{n}} X_{\mathbf{i}}$.

Proof Since NA implies pairwise NQD and for $\alpha=1$ the multiindex version of the Marcinkiewicz-Zygmund strong law for identically distributed NQD random variables was proved by Matula(see Matula, 1992, Theorem 2) we only need prove (2.2) for $\frac{1}{2}<\alpha<1$. We truncate at the level $|\mathbf{n}|^{\alpha}$ and set

$$
Y_{\mathbf{n}}=-|\mathbf{n}|^{\alpha} I\left[X_{\mathbf{n}}<-|\mathbf{n}|^{\alpha}\right]+X_{\mathbf{n}} I\left[\left|X_{\mathbf{n}}\right| \leq|\mathbf{n}|^{\alpha}\right]+|\mathbf{n}|^{\alpha} I\left[X_{\mathbf{n}}>|\mathbf{n}|^{\alpha}\right] .
$$

According to the property of NA random variables in Joag-Dev and Proschan (1983) $Y_{\mathbf{n}}$ 's are still identically distributed NA random variables.

Assume that (2.1) holds for $\frac{1}{2}<\alpha<1$. Then

$$
\begin{aligned}
\sum_{\mathbf{n}} P\left(X_{\mathbf{n}} \neq Y_{\mathbf{n}}\right) & =\sum_{\mathbf{n}} P\left(\left|X_{\mathbf{1}}\right|>|\mathbf{n}|^{\alpha}\right) \\
& =\sum_{\nu=1}^{\infty} P\left(\left|X_{\mathbf{1}}\right|>\nu^{\alpha}\right) \sum_{\nu=|\mathbf{n}|} 1 \\
& =\sum_{\nu=1}^{\infty} P\left(\left|X_{\mathbf{1}}\right|>\nu^{\alpha}\right) \Delta g(\nu) \\
& =\sum_{\nu=0}^{\infty} g(\nu) P\left(\nu^{\alpha}<\left|X_{\mathbf{1}}\right| \leq(\nu+1)^{\alpha}\right) \\
& \leq c \sum_{\nu=0}^{\infty} \frac{\nu(\log \nu)^{d-1}}{(d-1) !} P\left(\nu^{\alpha}<\left|X_{\mathbf{1}}\right| \leq(\nu+1)^{\alpha}\right) \\
& \leq c E\left(\left|X_{\mathbf{1}}\right|^{\frac{1}{\alpha}}\left(\log ^{+}\left|X_{\mathbf{1}}\right|\right)^{d-1}\right)<\infty \text { by }(2.1),
\end{aligned}
$$

where

$$
g(\nu):=\sum_{|\mathbf{n}| \leq \nu} \mathbf{1} \sim c \frac{\nu(\log \nu)^{d-1}}{(d-1) !} \text { as } \nu \rightarrow \infty
$$

with a suitable positive constant $c$ and $\Delta g(\nu)=g(\nu)-g(\nu-1)$.

Hence $P\left(X_{\mathbf{n}} \neq Y_{\mathbf{n}}\right.$, i.o. $)=0$ by the Borel-Cantelli lemma. 
Thus $\sum_{\mathbf{n}} \frac{X_{\mathbf{n}}}{|\mathbf{n}|^{\alpha}}$ converges almost surely if and only if $\sum_{\mathbf{n}} \frac{Y_{\mathbf{n}}}{|\mathbf{n}|^{\alpha}}$ converges almost surely. According to the multiindex Kolmogorv's convergence criterion(see, e.g. Gabriel(1977)), to prove $\sum_{\mathbf{n}} \frac{Y_{\mathbf{n}}}{|\mathbf{n}|^{\alpha}}$ converges almost surely we need to show that

$$
\sum_{\mathbf{n}} \frac{\left(Y_{\mathbf{n}}-E Y_{\mathbf{n}}\right)}{|\mathbf{n}|^{\alpha}} \text { converges almost surely }
$$

and

$$
\sum_{\mathbf{n}} \frac{E Y_{\mathbf{n}}}{|\mathbf{n}|^{\alpha}} \text { converges almost surely. }
$$

To prove (2.4), it is suffices to show that

$$
\operatorname{Var}\left(\sum_{\mathbf{n}} \frac{Y_{\mathbf{n}}}{|\mathbf{n}|^{\alpha}}\right)<\infty \text { as } \mathbf{n} \rightarrow \infty
$$

Since $Y_{\mathbf{n}}$ 's are identically distributed NA random variables we obtain

$$
\text { (2.7) } \begin{aligned}
\operatorname{Var}\left(\sum_{\mathbf{n}} \frac{Y_{\mathbf{n}}}{|\mathbf{n}|^{\alpha}}\right) & \leq \sum_{\mathbf{n}} \frac{\operatorname{Var}\left(Y_{\mathbf{n}}\right)}{|\mathbf{n}|^{2 \alpha}} \leq \sum_{\mathbf{n}} \frac{E Y_{\mathbf{n}}^{2}}{|\mathbf{n}|^{2 \alpha}} \\
& =\sum_{\mathbf{n}} P\left(X_{\mathbf{n}} \neq Y_{\mathbf{n}}\right)+\sum_{\mathbf{n}} \frac{E X_{1}^{2} I\left[\left|X_{\mathbf{1}}\right| \leq|\mathbf{n}|^{\alpha}\right]}{|\mathbf{n}|^{2 \alpha}} \\
& =I+I I .
\end{aligned}
$$

It is clear that $I<\infty$ by $(2.3)$. 
Using the function $f(\nu):=\sum_{|\mathbf{n}| \leq \nu} \mathbf{1} \sim \frac{\nu(\log \nu)^{d-1}}{(d-1) !}$ as $\nu \rightarrow \infty$ with differences $\Delta f=f(\nu)-f(\nu-1)=\sum_{|\mathbf{n}|=\nu} \mathbf{1}$, we estimate that

$$
\begin{aligned}
(2.8) I I & =\sum_{\nu=1}^{\infty} \Delta f(\nu) \frac{1}{\nu^{2 \alpha}} \sum_{j=1}^{\nu^{\alpha}} E X_{\mathbf{1}}^{2} 1\left[j-1<\left|X_{\mathbf{1}}\right| \leq j\right] \\
& \leq \sum_{\nu=1}^{\infty} \Delta f(\nu) \frac{1}{\nu^{2 \alpha}} \sum_{j=1}^{\nu^{\alpha}} j^{2} P\left[j-1<\left|X_{\mathbf{1}}\right| \leq j\right] \\
& \leq c \sum_{\nu=1}^{\infty} \frac{1}{\nu^{2 \alpha}} \Delta f(\nu) \sum_{i=1}^{\nu^{\alpha}} i P\left[\left|X_{\mathbf{1}}\right|>i\right] \\
& \leq c \sum_{i=1}^{\infty} i P\left[\left|X_{\mathbf{1}}\right|>i\right] \sum_{\nu=i \frac{1}{\alpha}}^{\infty} \nu^{-2 \alpha} \Delta f(\nu) \\
& \leq c \sum_{i=1}^{\infty} i^{\frac{1}{\alpha}-1}(\log i)^{d-1} P\left[\left|X_{\mathbf{1}}\right|>i\right] \\
& \leq c \sum_{i=1}^{\infty} i^{\frac{1}{\alpha}}(\log i)^{d-1} P\left[i-1<\left|X_{\mathbf{1}}\right| \leq i\right] \\
& \leq c E\left|X_{\mathbf{1}}\right|^{\frac{1}{\alpha}}\left(\log { }^{+}\left|X_{\mathbf{1}}\right|\right)^{d-1}<\infty \text { by }(2.1) .
\end{aligned}
$$

So (2.4) holds. To prove (2.5) it is sufficient to show that

$$
\begin{aligned}
& \left|\sum_{\mathbf{n}} \frac{E\left(Y_{\mathbf{n}}\right)}{|\mathbf{n}|^{\alpha}}\right| \\
\leq & \left|\sum_{\mathbf{n}} \frac{E\left(X_{1} 1\left[\left|X_{\mathbf{1}}\right| \leq|\mathbf{n}|^{\alpha}\right]\right)}{|\mathbf{n}|^{\alpha}}\right|+\sum_{\mathbf{n}} P\left(X_{\mathbf{n}} \neq Y_{\mathbf{n}}\right) \\
= & I I I+I<\infty .
\end{aligned}
$$


Clearly $I<\infty$ by (2.3). From (2.1) we have

$$
\begin{aligned}
I I I & =\left|\sum_{\mathbf{n}} \frac{-E\left(X_{1} 1\left[\left|X_{\mathbf{1}}\right|>|\mathbf{n}|^{\alpha}\right]\right)}{|\mathbf{n}|^{\alpha}}\right| \\
& \leq \sum_{\nu=1}^{\infty} \frac{1}{\nu^{\alpha}} \Delta g(\nu) \sum_{j=\nu}^{\infty} j^{\alpha} P\left((j-1)^{\alpha}<\left|X_{\mathbf{1}}\right| \leq j^{\alpha}\right) \\
& \leq \sum_{j=1}^{\infty} j^{\alpha} P\left((j-1)^{\alpha}<\left|X_{\mathbf{1}}\right| \leq j^{\alpha}\right) \sum_{\nu=1}^{j} \frac{1}{\nu^{\alpha}} \Delta g(\nu) \\
& \leq \sum_{\nu=0}^{\infty} g(\nu) P\left(\nu^{\alpha}<\left|X_{\mathbf{1}}\right|<(\nu+1)^{\alpha}\right) \\
& \leq c \sum_{\nu=0}^{\infty} \frac{\nu(\log \nu)^{d-1}}{(d-1) !} P\left(\nu^{\alpha}<\left|X_{\mathbf{1}}\right| \leq(\nu+1)^{\alpha}\right) \\
& \leq c E\left|X_{\mathbf{1}}\right|^{\frac{1}{\alpha}}\left(\log ^{+}\left|X_{\mathbf{1}}\right|\right)^{d-1}<\infty,
\end{aligned}
$$

which yields (2.9). So (2.5) holds.

Hence by $(2.4),(2.5)$ and Kolmogorov's convergence criterion $\sum_{\mathbf{n}} \frac{Y_{\mathbf{n}}}{|\mathbf{n}|^{\alpha}}$ converges almost surely, and thus $\sum_{\mathbf{n}} \frac{X_{\mathbf{n}}}{|\mathbf{n}|^{\alpha}}$ converges almost surely. Finally, by the multiindex Kronecker lemma (cf. Moore(1966) for the necessary multiindex partial sum formula) (2.2) holds.

For the converse, we note that (2.2) implies $\frac{X_{\mathbf{n}}}{|\mathbf{n}|^{\alpha}} \rightarrow 0$ as $\mathbf{n} \rightarrow \infty$ and $\frac{X_{\mathbf{n}}}{|\mathbf{n}|^{\alpha}} \rightarrow 0$ implies $\sum_{\mathbf{n}} P\left(\left|X_{\mathbf{1}}\right|>|\mathbf{n}|^{\alpha}\right)<\infty$, which yields that the desired moment condition (2.1) holds and obviously, that $E X_{1}=0$.

Corollary $2.2(\operatorname{Ko}(\mathbf{2 0 1 1}))$ Let $\left\{X_{\mathbf{n}}, \mathbf{n} \in \mathbb{Z}_{+}^{d}\right\}$ be a field of identically distributed NA random variables. Then

$$
E\left|X_{1}\right|\left(\log ^{+}\left|X_{1}\right|\right)^{d-1}<\infty \text { and } E X_{1}=0
$$

if and only if

$$
\frac{S_{\mathbf{n}}}{|\mathbf{n}|} \rightarrow 0 \text { a.s. } \mathbf{n} \rightarrow \infty
$$

Theorem 2.3 Let $\left\{X_{\mathbf{i}}, i \in \mathbb{Z}_{+}^{d}\right\}$ be a field of identically distributed NA random variables with $E X_{1}=0$ and $E X_{1}^{2}<\infty$. Then, for $\frac{1}{2}<\alpha<1$

$$
\frac{S_{\mathbf{n}}}{|\mathbf{n}|^{\alpha}} \rightarrow 0 \text { in } L^{\frac{1}{\alpha}} \text { and in probability a.s. } \mathbf{n} \rightarrow \infty \text {. }
$$


Proof for $\frac{1}{2}<\alpha$

$$
\left.E\left(\frac{\left|S_{\mathbf{n}}\right|}{|\mathbf{n}|^{\alpha}}\right)^{\frac{1}{\alpha}} \leq \frac{E S_{\mathbf{n}}^{2}}{|\mathbf{n}|^{2 \alpha}}\right)^{\frac{1}{2 \alpha}} \leq\left(\frac{|\mathbf{n}| E X_{\mathbf{1}}^{2}}{|\mathbf{n}|^{2 \alpha}}\right)^{\frac{1}{2 \alpha}} \rightarrow 0 \text { as }|\mathbf{n}| \rightarrow \infty,
$$

which establishes $L^{\frac{1}{\alpha}}$-convergence and hence, in particular, also convergence in probability.

Theorem 2.4 Let $\left\{X_{\mathbf{i}}, \mathbf{i} \in \mathbb{Z}_{+}^{d}\right\}$ be a field of identically distributed NA random variables with mean zero and finite variances and let $\frac{1}{2}<\alpha<1$. If

$$
|\mathbf{n}| P\left(\left|X_{1}\right|>|\mathbf{n}|^{\alpha}\right) \rightarrow 0 \text { as } \mathbf{n} \rightarrow \infty,
$$

then

$$
\frac{S_{\mathbf{n}}}{|\mathbf{n}|^{\alpha}} \rightarrow^{p} 0 \text { as } \mathbf{n} \rightarrow \infty
$$

where $\rightarrow^{p}$ means convergence in probability and $S_{\mathbf{n}}=\sum_{\mathbf{1} \leq \mathbf{k} \leq \mathbf{n}} X_{\mathbf{k}}$.

Proof Define $Y_{\mathbf{k}}^{\mathbf{n}}$, for $\mathbf{k} \leq \mathbf{n}$

$$
Y_{\mathbf{k}}^{\mathbf{n}}=-|\mathbf{n}|^{\alpha} I\left[X_{\mathbf{k}}<-|\mathbf{n}|^{\alpha}\right]+X_{\mathbf{k}} I\left[\left|X_{\mathbf{k}}\right| \leq|\mathbf{n}|^{\alpha}\right]+|\mathbf{n}|^{\alpha} I\left[X_{\mathbf{k}}>|\mathbf{n}|^{\alpha}\right]
$$

and $T_{\mathbf{n}}=\sum_{\mathbf{1} \leq \mathbf{k} \leq \mathbf{n}} Y_{\mathbf{k}}^{\mathbf{n}}$. Clearly, $X_{\mathbf{k}}-Y_{\mathbf{k}}^{\mathbf{n}}$ 's are NA random variables. Then, by assumptions (2.13) and $E X_{1}^{2}<\infty$ and Chebyshev's inequality we obtain

$$
\begin{aligned}
P\left(\frac{\left|S_{\mathbf{n}}\right|}{|\mathbf{n}|^{\alpha}}>\epsilon\right) \leq & P\left(\frac{\left|S_{\mathbf{n}}-T_{\mathbf{n}}\right|}{|\mathbf{n}|^{\alpha}}>\frac{\epsilon}{2}\right)+P\left(\frac{\left|T_{\mathbf{n}}\right|}{|\mathbf{n}|^{\alpha}}>\frac{\epsilon}{2}\right) \\
\leq & \frac{4 E\left(S_{\mathbf{n}}-T_{\mathbf{n}}\right)^{2}}{|\mathbf{n}|^{2 \alpha} \epsilon^{2}}+\frac{4 \sum_{\mathbf{1} \leq \mathbf{k} \leq \mathbf{n}} E X_{\mathbf{k}}^{2}}{|\mathbf{n}|^{2 \alpha} \epsilon^{2}} \\
\leq & \frac{4 \sum_{\mathbf{1} \leq \mathbf{k} \leq \mathbf{n}} E\left(X_{\mathbf{k}}-Y_{\mathbf{k}}^{\mathbf{n}}\right)^{2}}{|\mathbf{n}|^{2 \alpha} \epsilon^{2}}+\frac{4|\mathbf{n}| E Y_{\mathbf{1}}^{\mathbf{n}}}{|\mathbf{n}|^{2 \alpha} \epsilon^{2}} \\
= & \frac{4|\mathbf{n}| E\left|X_{\mathbf{1}}\right|^{2} I\left[\left|X_{\mathbf{1}}\right|>|\mathbf{n}|^{\alpha}\right]}{|\mathbf{n}|^{2 \alpha} \epsilon^{2}}+\frac{4|\mathbf{n}| E\left|X_{\mathbf{1}}\right|^{2} I\left[\left|X_{\mathbf{1}}\right| \leq|\mathbf{n}|^{\alpha}\right]}{|\mathbf{n}|^{2 \alpha} \epsilon^{2}} \\
& +\frac{\left.4|\mathbf{n}| \mathbf{n}\right|^{2 \alpha} P\left(\left|X_{\mathbf{1}}\right|>|\mathbf{n}|^{\alpha}\right)}{|\mathbf{n}|^{2 \alpha} \epsilon^{2}} \\
= & \frac{4|\mathbf{n}| E\left(X_{1}^{2}\right)}{|\mathbf{n}|^{2 \alpha} \epsilon^{2}}+4 \epsilon^{-2}|\mathbf{n}| P\left(\left|X_{\mathbf{1}}\right|>|\mathbf{n}|^{\alpha}\right) \rightarrow 0 \text { as } \mathbf{n} \rightarrow \infty .
\end{aligned}
$$

Hence, the proof is complete. 


\section{References}

[1] Etemati, N.(1981) An elementary proof of the strong law of large numbers, Z. Wahrsch. Verw. Gebiete 55 119-122

[2] Gabriel, J-P.(1977) An inequality for sums of independent random variables indexed by finite dimensional filtering sets and applications to the convergence of series, Ann. Probab. 5 779-786

[3] Gut, A.(1978) Marcinkiewicz laws and convergence rates in the law of large numbers for random variables with multidimensional indices, Ann. Probab. 6 469-482

[4] Gut, A.(1980) Convergence rates for probabilities of moderate deviations for sums of random variables with multidimensional indices, Ann. Probab. 8 298-313

[5] Gut, A. and Spătăru, A.(2003) Precise asymptotics in some strong limit theorems for multidimensionally indexed random variables, J. Multi. Anal. 86 398-442

[6] Hardy, G.H. and E.M. Wright(1954) An Introduction to the Theory of Numbers, 3rd Edition, Oxford University Press, Oxford

[7] Joag-Dev, K. and F. Proschan(1983) Negative association of random variables with applications, Ann. Statist. 11 286-295

[8] Ko, M.H.(2011) On the complete convergence for negatively associated random fields, Taiwan J. Math. 15 171-179

[9] Lehmann, E.L.(1966) Some concepts of dependence, Ann. Math. Statist. 37 11371153

[10] Matula, P.(1992) A note on the almost sure convergence of sums of negatively dependent random variables, Statist. Probab. Lett. 15 209-213

[11] Moore, C.N.(1966) Summable Series and Convergence Factors, Dover, New York

[12] Titchmarsh, E.C.(1951) The Theorey of the Rieman Zeta-Function, Oxford University Press, Oxford

\section{Mi-Hwa Ko}

Department of Mathematics, WonKwang University, Iksan, Jeonbuk 570-749, Korea.

E-mail: songhack@wonkwang.ac.kr 